\title{
Evidence of Two Distinct Dynamic Critical Exponents in Connection with Vortex Physics
}

\author{
Petter Minnhagen and Beom Jun Kim \\ Department of Theoretical Physics, Umeå University, 90187 Umeå, Sweden \\ Hans Weber \\ Department of Physics, Luleå University of Technology, 97187 Luleå, Sweden
}

(Received 1 February 2001; published 29 June 2001)

\begin{abstract}
The dynamic critical exponent $z$ is determined from numerical simulations for the three-dimensional (3D) lattice Coulomb gas (LCG) and the 3D $X Y$ models with relaxational dynamics. It is suggested that the dynamics is characterized by two distinct dynamic critical indices $z_{0}$ and $z$ related to the divergence of the relaxation time $\tau$ by $\tau \propto \xi^{z_{0}}$ and $\tau \propto k^{-z}$, where $\xi$ is the correlation length and $k$ the wave vector. The values determined are $z_{0} \approx 1.5$ and $z \approx 1$ for the 3D LCG and $z_{0} \approx 1.5$ and $z \approx 2$ for the 3D $X Y$ model. Comparisons with other results are discussed.
\end{abstract}

DOI: $10.1103 /$ PhysRevLett.87.037002

A neutral superfluid such as ${ }^{4} \mathrm{He}$ and a superconductor in the limit of large London penetration depth can be characterized by a complex order parameter. The $X Y$ model can be viewed as a discretized version of this type of system in terms of the phase of the order parameter [1]. An interesting feature of this class of models is the presence of thermally generated topological defects which in three dimensions (3D) take the form of vortex loops. The superconducting phase transition from the vortex loop point of view separates a low-temperature phase consisting of closed vortex loops of finite extent from a high-temperature phase where the loops can disintegrate [2-4]. The static thermodynamic properties of the $X Y$ model allow a dual representation in terms of the vortex degrees of freedom. In particular, the Villain transformation of the $X Y$ model gives rise to the dual vortex loop model termed the lattice Coulomb gas (LCG) [5]. A crucial point in the following is that this duality does not include the dynamic properties which might be quite different.

The universality of the dynamic behavior is weaker and requires in addition to the static universality also that certain global features of the dynamics are the same. In the dynamic universality classes defined by Hohenberg and Halperin [6], these additional global features are expressed as local conservation laws. According to this scheme, the dynamics of a 3D superfluid belongs to model $\mathrm{F}$ characterized by the dynamic critical exponent $z=1.5$ [7]. A model with purely relaxational dynamics on the other hand belongs to model A with $z \approx 2$ [6]. In case of a superconductor, both model $\mathrm{F}$ and model $\mathrm{A}$ have been proposed as the appropriate dynamical class $[6,8]$. Since relaxational dynamics is related to model A, it might appear surprising that the critical dynamic index $z$ for the 3D LCG for periodic boundary conditions (PBC) and with purely relaxational dynamics in Ref. [9] (verified in Ref. [10]) was found to be $z \approx 1.5$ instead of $z \approx 2$. Similarly, the 3D $X Y$ model with fluctuating twist boundary condition (FTBC) and relaxational dynamics was found to have $z \approx 1.5$ [11]. On the other hand, the 3D $X Y$ model with
PACS numbers: 74.60.-w, 05.70.Jk, 75.40.Gb, 75.40.Mg

$\mathrm{PBC}$ and relaxational dynamics has $z \approx 2$ consistent with model A. This implies that the choice of boundary condition affects the value of $z$ [11]. In the present Letter, we show that the same type of sensitivity applies to the 3D LCG, giving rise to two distinct exponents $z \approx 1.5$ and $z \approx 1$. It is proposed that these results for the LCG model and the $X Y$ model reflect the existence of two distinct critical indices corresponding to $\tau \propto \xi^{z_{0}}$ and $\tau \propto k^{-z}$, where $\tau, \xi$, and $k$ denote relaxation time, coherence length, and wave vector, respectively.

The 3D LCG on a cubic lattice is defined by the Hamiltonian $[5,12,13]$,

$$
H=\frac{1}{2} \sum_{\alpha=1}^{3} \sum_{i, j} q_{\alpha i} W\left(\mathbf{r}_{i}-\mathbf{r}_{j}\right) q_{\alpha j}
$$

where $\mathbf{q}_{i} \equiv\left(q_{\alpha i}\right)$ represent the vortex line segment variables for each lattice site, one for each of the three lattice directions $\mathbf{e}_{\alpha}, q_{\alpha i} \in[0,1,-1]$ corresponding to no vortex segment, respectively, to a segment with vorticity 1 or -1 between neighboring lattice sites and subject to the constraint that the sum of the $q_{\alpha i}$ 's for the six directed links reaching each lattice site is zero. We use $\mathrm{PBC}$ and $W(r)$ is the lattice version of the Coulomb interaction [13],

$$
W(\mathbf{r})=\frac{1}{L^{3}} \sum_{\mathbf{k}} \frac{4 \pi^{2} e^{i \mathbf{k}} \cdot \mathbf{r}}{\boldsymbol{\kappa}^{2}(\mathbf{k})},
$$

where $L$ is the total length of the lattice, the lattice constant is 1 , and $\kappa_{\alpha}=2 \sin \left(k_{\alpha} / 2\right)$, where $\alpha=1,2$, and 3 . The relaxational dynamics is implemented by a Metropolis Monte Carlo update where each complete update of the lattice is associated with one time unit (for details see Ref. [9]). The voltage across the sample $\mathbf{V}$ is proportional to the expansion rate of the vortex loops $\mathbf{V} \propto \frac{d}{d t} \sum_{\mathbf{r}} \mathbf{r} \times$ $\mathbf{q}_{\mathbf{r}}$, where $\mathbf{r}$ denotes the site positions on the lattice. The resistance $R$ can then be obtained from the voltage fluctuations through the Nyquist formula $R \propto \int_{-\infty}^{+\infty} d t\langle V(t) V(0)\rangle$. The scaling connection, $R \propto 1 / \tau$, leads to the size scaling at $T_{c}, R \propto L^{-z_{0}}$, and the $\xi$ scaling of the resistivity, $\rho \propto \xi^{1-z_{0}}$ in the critical region [8]. The 3D LCG has 
a phase transition at $T_{c} \approx 3.003$ with $\nu \approx 0.67$ [14,15]. Finite-size scaling at $T_{c}$ gives $z_{0} \approx 1.5$ [9,10]. In Fig. 1, we demonstrate that the size converged $\xi$ scaling slightly above $T_{c}$, using $\xi \propto\left(T-T_{c}\right)^{-\nu}$, also gives $z_{0} \approx 1.5$. This shows that $z_{0} \approx 1.5$ is not a finite-size effect caused by the boundary, but is a bulk property characterizing the dynamics.

The LCG model defined with periodic boundary conditions (PBC) corresponds to the $X Y$ model defined with the fluctuating twist boundary condition (FTBC) [16]. The Hamiltonian for the latter model is given by

$$
H\left(\theta_{\mathbf{r}}, \boldsymbol{\Delta}\right)=-\sum_{\mathbf{r} \alpha} \cos \left(\theta_{\mathbf{r}}-\theta_{\mathbf{r}+\alpha}-\mathbf{e}_{\alpha} \cdot \mathbf{\Delta}\right),
$$

where the $\alpha$ summation is over the three nearest neighbors of $\mathbf{r}$ in the three lattice base directions. The relaxational dynamics is, as for the LCG model, implemented by a Metropolis Monte Carlo update where each complete update of the lattice is associated with one time unit (for details see Ref. [17]). We use PBC for $\theta_{\mathbf{r}}$ which means that $\Delta_{\alpha}$ is the average twist of the angle $\theta(\mathbf{r})$ across the lattice in the $\alpha$ direction. The fluctuations in the twist variable $\Delta_{\alpha}(t)$ are directly related to the voltage $V_{\alpha}$ across the sample by $V_{\alpha}=-L \frac{d}{d t} \Delta_{\alpha}$ [18]. The correspondence between LCG with PBC and the $X Y$ model with FTBC basically hinges on the fact that the models defined with

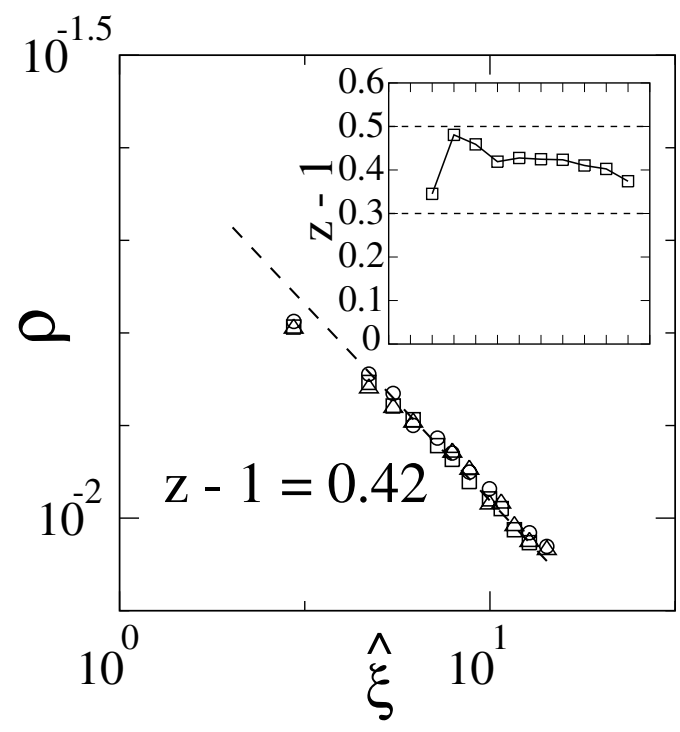

FIG. 1. Determination of $z_{0}$ from $\rho \propto \xi^{1-z_{0}}$ for the 3D LCG with PBC for lattice sizes $L=10,12$, and 16 (triangles, circles, and squares, respectively) and $\xi \propto \hat{\xi} \equiv\left(T-T_{c}\right)^{-\nu}$ (see text). As seen, the data are independent of lattice size. The broken line is a least square fit to the linear part of the data and gives $z_{0} \approx 1.4(1)$ consistent with $z_{0} \approx 1.5$. The inset shows $z-1$ from the slope $(z-1=-$ slope $)$ for the $L=16$ data points, obtained by least square fitting starting from the two data points for the largest $\hat{\xi}$ and then consecutively adding more data points so that the rightmost data point in the inset is based on all but one data point in the main figure. The dashed lines in the inset give our rough estimate of the error. these boundary conditions allow for voltage fluctuations across the system. In contrast, the usual PBC applied to the $3 \mathrm{D} X Y$ model is equivalent to $\boldsymbol{\Delta} \equiv 0$ and the voltage fluctuations across the system are then no longer properly described by the model [11]. The 3D $X Y$ model with PBC, within the Villain approximation, corresponds to the $3 \mathrm{D}$ LCG model with PBC described by the modified Hamiltonian [19] [compare Eq. (1)],

$$
H=\sum_{\alpha=1}^{3}\left[\frac{1}{2} \sum_{i, j} q_{\alpha i} W\left(\mathbf{r}_{i}-\mathbf{r}_{j}\right) q_{\alpha j}+U\left(2 \pi M_{\alpha} / L^{2}\right)\right],
$$

where $U(\phi)=\sum_{m=-\infty}^{\infty} \exp \left[-(\phi+2 m)^{2} / 2 T\right]$ is the Villain function, and $M_{\alpha}$ is given by $\mathbf{M}=\frac{1}{2} \sum_{\mathbf{r}} \mathbf{r} \times \mathbf{q}_{\mathbf{r}}$. Since this Hamiltonian in Fourier space has the structure $H \propto \sum_{\mathbf{k} \neq 0, \alpha} \mathbf{q}_{\alpha}(\mathbf{k}) W(\mathbf{k}) \mathbf{q}_{\alpha}(-\mathbf{k})+\delta_{\mathbf{k} 0} U$, the difference with Eq. (1) is that in the modified Hamiltonian (4) the $k=0$ mode is suppressed. We focus on the scaling of the vorticity correlation function,

$$
G(k, t)=\frac{1}{\mathbf{k}^{2} L^{3}}\left\langle q_{\alpha \mathbf{k}}(t) q_{\alpha-\mathbf{k}}(0)\right\rangle,
$$

where $\mathbf{k}$ is perpendicular to the $\alpha$ direction. In particular, $G(k=0, t)$ has the scaling form $G(k=0, t) \propto$ $F\left(t \xi^{-z}\right) / \xi$ [11]. Figure 2 demonstrates that this scaling is well borne out with the value $z \approx 1$. The data are from the critical region just above $T_{c}$ where the data are size converged and $\xi \propto\left(T-T_{c}\right)^{-\nu}$, which we again emphasize

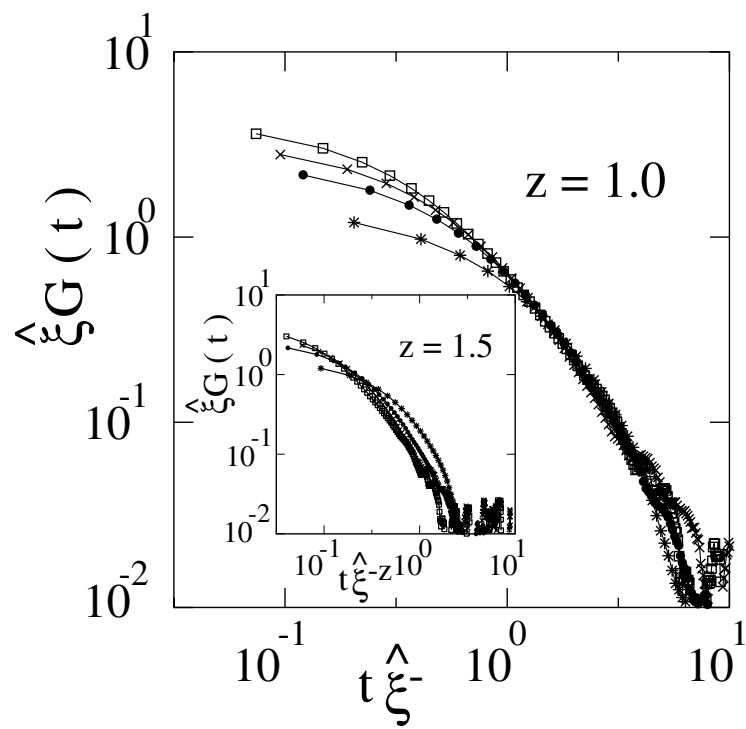

FIG. 2. Determination of $z$ from the scaling $\xi G(0, t) \propto$ $F\left(t \xi^{-z}\right)$ for the modified 3D LCG (corresponding to the 3D $X Y$ model with PBC). The data are for lattice size $L=20$ and $T=3.07,3.10,3.14$, and 3.30 (open squares, crosses, solid circles, and asterisks, respectively). A good collapse is obtained for $z \approx 1$, whereas the inset shows that no collapse is obtained for $z=1.5$ ( $\hat{\xi}$ is defined as in Fig. 1 ). Consequently, $z \approx 1$, which is different from $z_{0} \approx 1.5$ obtained in Fig. 1 . 
means that $z \approx 1$ is a bulk property and not a property that vanishes for $L=\infty$. Thus, just as for the 3D $X Y$ model in Ref. [11], we obtain two distinct values of $z \approx 1.5$ and $z \approx 1$, by allowing and suppressing the $k=0$ mode.

As a resolution of this dichotomy, we suggest that these models are characterized by two distinct indices corresponding to $\tau \propto \xi^{z_{0}}$ and $\tau \propto k^{-z}$. Assuming such a resolution would mean that the vorticity correlation function $G(k, t)$ in Eq. (5) in general scales as $G(k, t) \propto$ $F\left(t k^{z}, t \xi^{-z_{0}}, k \xi\right) / \xi$, which precisely at $T_{c}$ for $k_{\min }=$ $2 \pi / L$ reduces to

$$
L G\left(k_{\min }, t\right)=F\left(t k_{\min }^{z}, t L^{-z_{0}}\right) .
$$

Choosing $t=x k_{\min }^{-z}$ gives $F\left(x, x L^{z-z_{0}}\right)$ which goes to $F(x, \infty)$ for large $L$ when $z>z_{0}$ and $F(x, 0)$ for $z<z_{0}$. This means that, in the scaling limit of large $t\left(t \gg \tau_{0}\right.$, where $\tau_{0}$ is a microscopic characteristic time), we will approach a scaling $\tilde{F}(x)$ with $x=t k_{\min }^{z}$ for large (small) $x$ when $z>z_{0}\left(z<z_{0}\right)$. Vice versa, we will approach a scaling limit $\hat{F}(x)$ with $x=t L^{-z_{0}}$ for large (small) $x$ when $z_{0}>z\left(z_{0}<z\right)$.

We first test the possibility of two distinct indices for the 3D $X Y$ model with FTBC [see Eq. (3)]. The resistance $R$ for the 3D $X Y$ with FTBC is readily calculated from $\Delta_{\alpha}(t)$ (see [18] for details). $G(k, t)$ is obtained by replacing $q_{\alpha \mathbf{r}}$ with $\tilde{q}_{\alpha}(\mathbf{r}) \equiv \sin \left(\theta_{\mathbf{r}}-\theta_{\mathbf{r}+\mathbf{e}_{\alpha}}\right)$ in Eq. (5) and multiplying by $k^{2}$ [20]:

$$
G(k, t)=\frac{1}{L^{3}}\left\langle\tilde{q}_{\alpha \mathbf{k}}(t) \tilde{q}_{\alpha-\mathbf{k}}(0)\right\rangle .
$$

Figures 3(a) and 3(b) demonstrate, by using the scaling form $R \propto L^{-z_{0}} f\left[L\left(T-T_{c}\right)^{-\nu}\right]$ and $L G(k, t)=\tilde{F}\left(t k_{\min }^{z}\right)$, that for this model $z_{0} \approx 1.5$ whereas $z \approx 2$, confirming that $z_{0} \neq z$. For $z_{0}<z$ it should, in principle, also be possible to observe a crossover to $\tilde{F}\left(t L^{-z_{0}}\right)$ for small enough arguments (but still with $t \gg \tau_{0}$ ); however, this limit was not reached in the simulations [21]. It is interesting to note that the exponent $z \approx 2$ associated with $\tau \propto k_{\min }^{z}$ for the 3D $X Y$ model with FTBC, within error bars, has the same value as the exponent $z_{0} \approx 2$ found for the 3D $X Y$ model with PBC [11]. This suggests that the difference between FTBC and PBC arises from the difference in the treatment of the voltage fluctuations across the system, or, equivalently, the $k=0$ fluctuations: The $k=0$ fluctuations for FTBC are associated with $z_{0} \approx 1.5$. Changing to PBC suppresses these fluctuations and the $k=0$ fluctuations for PBC would then correspond to the $k_{\min }=2 \pi / L$ fluctuations for FTBC. This argument implies that $z_{0}$ for PBC and $z$ for FTBC should be identical, in accordance with our numerical data.

Applying the same reasoning to the 3D LCG with PBC would mean that $z_{0} \approx 1.5$ and $z \approx 1$. Since in this case $z<z_{0}$, this would mean that $L G\left(k_{\min }, t\right)$ should scale similar to $\tilde{F}(x)$ with $x=k_{\min }^{z} t$ for small enough $x$ [compare Eq. (6)]. Figure 4(a) shows that this prediction is consistent with the data and that the scaling curve approaches
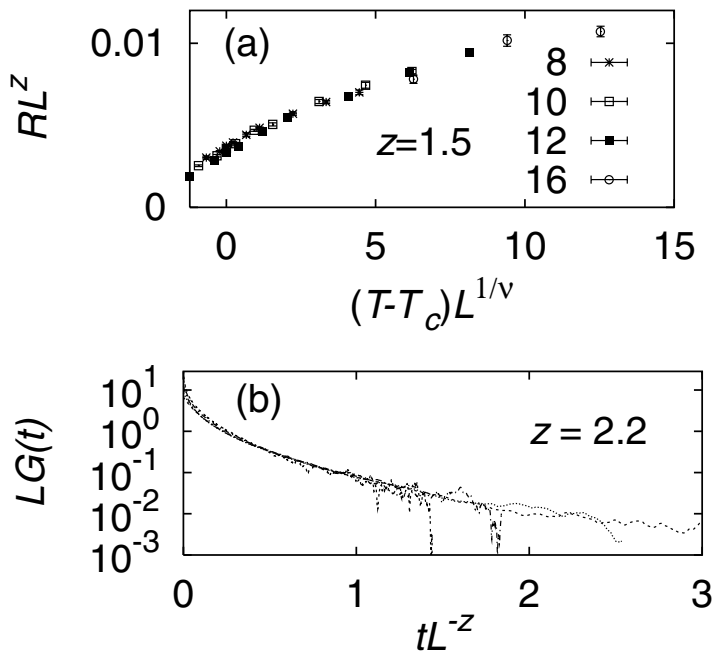

FIG. 3. Demonstration that the 3D $X Y$ model with FTBC contains two distinct indices $z$ and $z_{0}$, where $z \neq z_{0}$. (a) Scaling of resistance, $R \approx L^{-z_{0}} \tilde{F}\left[L\left(T-T_{c}\right)^{-\nu}\right]$, close to and at $T_{c}$ gives $z_{0} \approx 1.5$. (b) Scaling $L G\left(k_{\min }, t\right)=F\left(t k_{\min }^{z}, t L^{-z_{0}}\right)$ at $T_{c}$ gives $z \approx 2$ for large $t(L=10,12,16)$. Note that the scaling with the larger of $z$ and $z_{0}$ always dominates in the large $t$ limit.

the correct asymptotic form $\tilde{F}(x) \propto 1 / x^{1 / z}[22]$ in the limit of small $x$. Figure 4(b) shows that the data for the largest converged $t$ values are instead consistent with the scaling $\tilde{F}\left(x=t L^{-z_{0}}\right)$, which is also in accord with the scaling form given by Eq. (6). From this we conclude that the apparent nonuniqueness of the critical exponent $z$ for the 3D $X Y$ model and the LCG with relaxational dynamics is consistent with the existence of two distinct critical indices $z_{0}$ and $z$ for the case when the models are defined so as to allow for voltage fluctuations across the system.

We have here proposed the existence of two indices for relaxational dynamics in 3D. On the other hand, the corresponding model with the resistively shunted Josephson junction dynamics (RSJ) has $z_{0}=z[11,22]$. This model is closely related to the $X Y$ model and differs in that the dynamics is subject to local current conservation, i.e., it has a local conservation law which is not fulfilled by relaxational dynamics. In 2D, the whole low-temperature phase below the Kosterlitz Thouless transition is quasicritical and for the 2D $X Y$ model (with FTBC) $z_{0}(T)=$ $2 \pi \Upsilon(T)-2>2$, where $Y$ is the helicity modulus, and this value has been confirmed both for relaxational and RSJ dynamics, whereas $z=2 \neq z_{0}$ was found for the same cases [18]. Consequently, the existence of two indices $z_{0} \neq z$ appears not to be restricted to relaxational dynamics. One may also observe that the $z$ value for the $X Y$ model with relaxational dynamics is consistent with the model A value $z \approx 2$ in the Hohenberg-Halperin classification scheme [6] which to us suggests that the $z$ defined from $\tau \propto k^{-z}$ will, in general, be consistent with this classification scheme.

A common measurement for high $T_{c}$ superconductors is the $I V$ characteristics. In this experiment, the voltage 

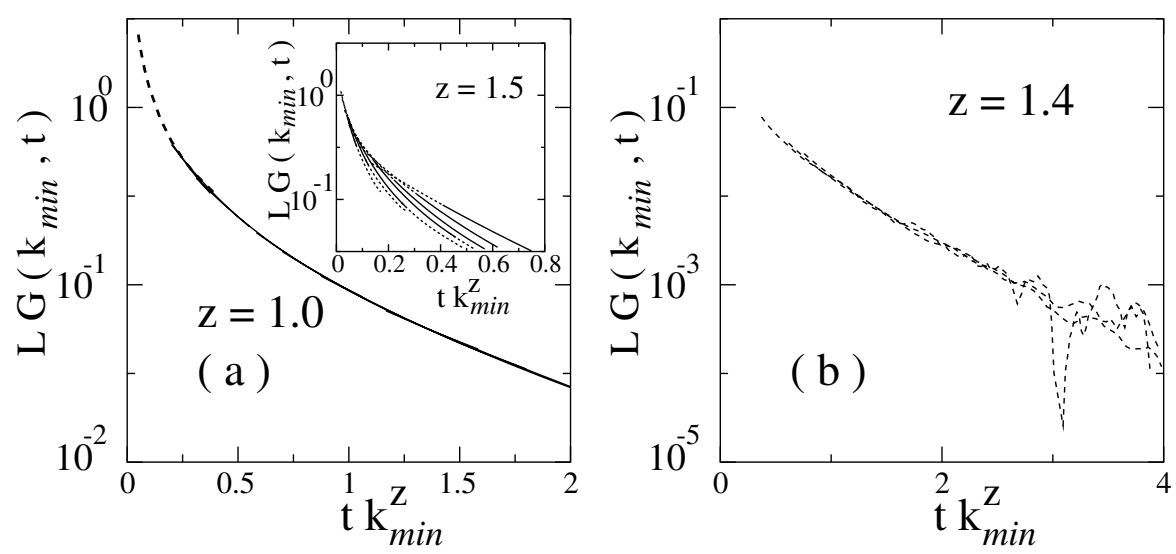

FIG. 4. Demonstration that the 3D LCG with PBC at $T_{c}$ contains two distinct indices $z$ and $z_{0}$ within the single scaling function $L G\left(k_{\min }, t\right)=F\left(t k_{\min }^{z}, t L^{-z_{0}}\right)$. (a) The data are for $L=6,8,10,12,16,20$, and 24 . The inset shows that $L G\left(k_{\min }, t\right)$ does not scale with $t k_{\min }^{z=1.5}$ for the data shown (the value of $L G$ decreases for increasing $L$ for a fixed $t k_{\min }^{z=1.5}$ ). The full drawn part of the data for each size in the inset gives a middle section of the data corresponding to neither too large nor too small values of $t$. These full drawn middle sections of the data in the inset are the parts that collapse to a single scaling curve for $z \approx 1$, as demonstrated by the main part of the figure. The broken curve in the main part is the leading small $t k_{\min }^{z}$ dependence of the scaling function given by $C / t k_{\min }^{z}$, where $C$ is a constant. This leading term is consistent with the scaling curve obtained from the data $(C$ is used as an adjustable parameter). (b) Data for $L=10,12$, and 16 obtained for larger $t$. These larger $t$ data collapse to a single curve with $z \approx 1.4$. Note that this is consistent with the fact that the larger of $z$ and $z_{0}$ dominates in the large $t$ limit.

across the sample is measured and, consequently, this measurement relates to $z_{0}$. The scaling prediction for the nonlinear $I V$ characteristics gives $V \propto I^{a}$, where $a=$ $\left(z_{0}+1\right) /(d-1)$ in $d$ dimensions [8]. A comparison with $a=(z+1) /(d-1)$, where $z$ is calculated according to the Hohenberg-Halperin classification scheme, is then likely to be incorrect if the superconductor is described by 3D model A (because $z_{0} \neq z$ for this case), but would be correct if it is described by 3D model $\mathrm{F}$ (because $z_{0}=z$ for this case $[11,22])$. Consequently, the possibility of the existence of two critical dynamic indices would have to be taken into account when analyzing experiments. A criterion for when $z \neq z_{0}$ and $z=z_{0}$ remains to be found.

[1] For a review, see, e.g., P. Minnhagen, Rev. Mod. Phys. 59, 1001 (1987).

[2] G. A. Williams, Phys. Rev. Lett. 82, 1201 (1999).

[3] G. A. Williams, Phys. Rev. Lett. 59, 1926 (1987); S. R. Shenoy, Phys. Rev. B 40, 5056 (1989); B. Chattopadhyay, M. C. Mahato, and S. R. Shenoy ibid. 47, 15159 (1993).

[4] G. A. Williams, Phys. Rev. Lett. 71, 392 (1993); J. Low Temp. Phys. 93, 1079 (1993).

[5] J. Villain, J. Phys. (Paris) 36, 581 (1975).

[6] P. C. Hohenberg and B. I. Halperin, Rev. Mod. Phys. 49, 435 (1977).

[7] The equality holds for a model where the critical exponent of the specific heat has a value $\alpha \leq 0$ as for the 3D XY model.
[8] D. S. Fisher, M. P. A. Fisher, and D. A. Huse, Phys. Rev. B 43, 130 (1991).

[9] H. Weber and H. J. Jensen, Phys. Rev. Lett. 78, 2620 (1997).

[10] J. Lidmar, M. Wallin, C. Wengel, S. M. Girvin, and A. P. Young, Phys. Rev. B 58, 2827 (1998).

[11] L. M. Melwyn, B. J. Kim, and P. Minnhagen, Europhys. Lett. 49, 644 (2000); Phys. Rev. B 61, 15412 (2000).

[12] J. V. José, L. P. Kadanoff, S. Kirkpatrick, and D. R. Nelson, Phys. Rev. B 16, 1217 (1977).

[13] G. Carneiro, Phys. Rev. B 45, 2391 (1992); G. Carneiro, R. Cavalcanti, and A. Gartner, Europhys. Lett. 11, 757 (1990); Phys. Rev. B 47, 5263 (1993).

[14] P. Olsson (unpublished).

[15] M. Hasenbusch and T. Tökök, J. Phys. A 32, 6361 (1999).

[16] P. Olsson, Phys. Rev. B 52, 4511 (1995).

[17] B. J. Kim, Phys. Rev. B 63, 024503 (2001).

[18] B. J. Kim, P. Minnhagen, and P. Olsson, Phys. Rev. B 59, 11506 (1999).

[19] A. Vallat and H. Beck, Phys. Rev. B 50, 4015 (1994); J. Lidmar and M. Wallin, Phys. Rev. B 59, 8451 (1999); S. Teitel (private communication).

[20] P. Minnhagen, in Lectures on Superconductivity in Networks and Mesoscopic Systems, edited by C. Giovannella and C. Lambert, AIP Conf. Proc. No. 427 (AIP, New York, 1998), p. 200.

[21] This limit simultaneously requires $t \gg \tau_{0}$ and $t L^{-z_{0}} \ll 1$. We believe that this requires converged data for larger $L$ than we could achieve.

[22] H. Weber, B. J. Kim, and P. Minnhagen (unpublished). 